
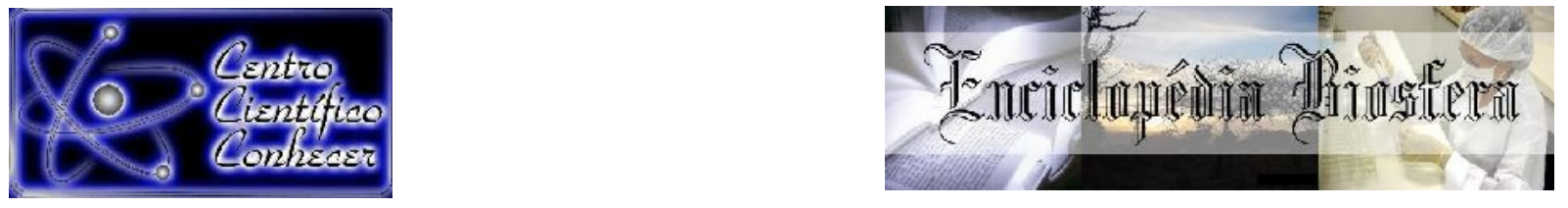

\title{
USO DE CINZA VEGETAL NO CULTIVO DE GLADÍOLOS
}

\author{
Ane Caroline Barceli ${ }^{1}$, Kassio dos Santos Carvalho ${ }^{2}$, Mayara Pontes Mariano ${ }^{1}$
}

1. Estudante do curso de Bacharelado em Engenharia Agronômica do Instituto Federal de Educação, Ciência e Tecnologia de Mato Grosso, campus Sorriso - Brasil

(anebarceli@gmail.com)

1. Estudante do curso de Bacharelado em Engenharia Agronômica do Instituto Federal de Educação, Ciência e Tecnologia de Mato Grosso, campus Sorriso - Brasil

2. Professor Doutor do Instituto Federal de Educação, Ciência e Tecnologia de Mato Grosso, campus Sorriso - Brasil

Recebido em: 15/11/2020 - Aprovado em: 15/12/2020 - Publicado em: 30/12/2020 DOI: 10.18677/EnciBio_2020D39

\begin{abstract}
RESUMO
O gladíolo é uma flor de corte utilizada para decoração de ambientes e o seu cultivo requer um solo com $\mathrm{pH}$ corrigido e fértil. Uma alternativa para aumentar o $\mathrm{pH}$ e o nível de fertilidade é o uso de cinza vegetal. Objetivou-se com o presente estudo avaliar o efeito de adubação com diferentes doses de cinza vegetal $\left(0,4,8,12,16 \mathrm{~g} / \mathrm{dm}^{3}\right)$ sobre o desempenho agronômico da cultura do gladíolos. A espécie estudada foi o Gladiolus $x$ grandiflorus e delineamento foi inteiramente casualizado com cinco tratamentos $\mathrm{e}$ seis repetições, totalizando 30 amostras. O crescimento e produção do gladíolo foram caracterizados pelas variáveis: altura de plantas, diâmetro do caule, comprimento da haste floral, altura da espiga, número de flores, massa seca de plantas. Os resultados foram submetidos à análise de variância até $5 \%$ de probabilidade pelo teste $\mathrm{F}$ e regressão.
\end{abstract}

PALAVRAS-CHAVE: Flores ornamentais, Gladiolus L., Resíduos Agroindustriais.

\section{USE OF VEGETABLE ASH IN THE CULTIVATION OF GLADIOLOS}

\section{ABSTRACT}

The gladiolus also known as Palm or Palm of Saint Rita is a cut flower used for decoration of environments, a solution for the use of waste is the use of vegetable ash from boiler burning can be a low cost alternative for fertilization of cover crops and green fertilizers that provides various nutrients. The purpose of this study was to evaluate the effect of fertilization with different doses of vegetable ash $(0,4,8,12,16$ $\mathrm{g} / \mathrm{dm}^{3}$ ) on the productive behavior of the crop in a protected environment. The species studied was Gladiolus x grandiflorus. The design was entirely randomized with 5 treatments and six repetitions, totaling 30 samples. Gladiolus growth and production were characterized by the following variables: number of leaves, diameter of stem total height of the plant, length of the ear, number of flowers, dry mass of the floral stem and total, number of days for spiking and flowering, number and weight of bulbs. The results were submitted to variance analysis up to $5 \%$ probability by $\mathrm{F}$ test and regression.

KEYWORDS: Ornamental flowers, Gladiolus L., Agro-industrial waste. 


\section{INTRODUÇÃO}

Gladiolus $x$ grandiflorus, popularmente conhecido como gladíolo ou palma-deSanta-Rita, é uma planta herbácea, pertencente à família Iridaceae, obtida pela hibridação entre diversas espécies nativas do sul do continente africano e do mediterrâneo. É uma planta heliófila, propagada vegetativamente por meio de bulbos. Pode ser cultivada ao longo de todo o ano, exceto em regiões que apresentem períodos com geadas. A inflorescência é do tipo espiga e se destaca como importante flor de corte no mercado mundial (AHMAD et al., 2008; AHMAD et al., 2011; SINGH et al., 2012).

Com a expansão do comércio de flores no Brasil, o gladíolo tornou-se uma das culturas mais expressivas, ocupando o quarto lugar em produção. Isso devido à facilidade de cultivo, caracterizada pela tolerância a pragas e doenças, ciclo curto, cultivo a céu aberto, produção de flores, bulbos e bulbilhos e à grande diversidade de coloração das flores, além da fácil condução, do baixo custo de implantação, do rápido retorno financeiro e possibilidade de cultivo em pequenas áreas (BARBOSA, 2011).

A aplicação de cinza vegetal em plantios agrícolas apresenta-se como uma importante oportunidade de restituição de parte dos nutrientes removidos pelas culturas, reduzindo a necessidade do uso de fertilizantes comerciais Santos (2012) e consequentemente os custos da produção, e contribuindo com a redução da acidificação (ZIMMERMANN; FREY, 2002). Diante disso, o seu aproveitamento na agricultura seria duplamente benéfico, por melhorar a produtividade das culturas e por minimizar o efeito poluente diante da elevada quantidade de cinzas produzidas (GUARIZ et al., 2009).Tendo em vista a geração em larga escala desse resíduo sólido pelo setor industrial, a destinação desse subproduto torna-se de fundamental importância. Uma alternativa que pode ser viável é o uso desse resíduo na produção de gladíolos. Dessa forma, objetivou -se avaliar o efeito de diferentes doses de cinza vegetal na produção de gladíolos.

\section{MATERIAL E MÉTODOS}

O experimento foi realizado no período de Março a Julho de 2019, em casa de vegetação no Instituto Federal de Educação, Ciência e Tecnologia de Mato Grosso IFMT, Campus Universitário de Sorriso, no Município de Sorriso-MT, localizado a 365

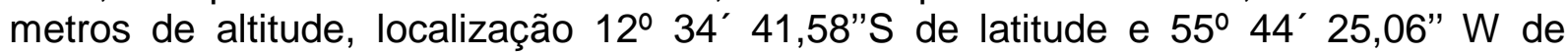
longitude.

O solo foi coletado na área experimental do Campus Universitário sendo classificado como LATOSSOLO Vermelho de textura argilosa, coletado na camada de 0,0-0,20 m, cuja caracterização química e granulométrica foi feita de acordo com EMBRAPA (2013). Além disso, o solo também foi passado em peneira de $4 \mathrm{~mm}$ antes de ser colocado nos vasos para a instalação do experimento, sendo posteriormente incubado com calcário dolomítico (PRNT = 80\%), elevando-se a saturação por bases para $70 \%$. 
TABELA 1. Caracterização química e granulométrica do LATOSSOLO Vermelho.

\begin{tabular}{|c|c|c|c|c|c|c|c|c|c|c|c|c|}
\hline $\mathrm{Ph}$ & P & $\mathrm{K}$ & $\mathrm{Ca}$ & $\mathrm{Mg} \quad \mathrm{Al}$ & $\mathrm{HCC}$ & СТC & M.O. & V & $\mathrm{m}$ & Areia & Silte & Argila \\
\hline $\mathrm{CaCl}_{2}{ }^{+}$ & mg.d & & & $\mathrm{cmol}_{\mathrm{c} \cdot \mathrm{C}}$ & $\mathrm{dm}_{3}$ & & g.kc & & & $\%$ & & g.kg ${ }^{1}$ \\
\hline 4,8 & 7,2 & 31,8 & 2,07 & 0,37 & 4,5 & 7 & 1,9 & 36 & 0 & 480 & 97 & 423 \\
\hline
\end{tabular}

TABELA 2. Caracterização química da cinza vegetal

\begin{tabular}{cccccc}
\hline P2O5 CNA + H20 & K2 O Sol em H20 & $\mathrm{Ca}(\%)$ & $\mathrm{Mg}(\%)$ & $\mathrm{S}-\mathrm{SO} 4(\%)$ & $\begin{array}{c}\text { metais pesados } \\
\text { micronutrientes }\end{array}$ \\
\hline 0,1 & 0,13 & 2,85 & 0,56 & $\mathrm{~ns}$ & $\mathrm{~ns}$ \\
\hline
\end{tabular}

O delineamento utilizado no trabalho foi inteiramente casualizado, com cinco tratamentos e seis repetições, totalizando 30 parcelas experimentais. Os tratamentos consistiram em doses de cinza vegetal: $0,4,8,12 \mathrm{e}_{16} \mathrm{~g} \mathrm{dm}^{-3}$. Cada parcela experimental foi constituída por um vaso com capacidade para $15 \mathrm{dm}^{3}$ de solo e com uma planta. As variáveis analisadas neste estudo foram:

- Diâmetro de caule e altura total da planta;

O diâmetro de caule e a altura total das plantas foram avaliados aos $30,45,60$ e 75 dias após o plantio (DAP), o diâmetro com auxílio de um paquímetro e altura com uma régua graduada que corresponde à altura da base da planta rente ao solo até a folha maior.

- Comprimento da espiga e haste floral;

A medição de comprimento da espiga e haste floral foi feita no ponto de colheita $(\mathrm{PC})$, com o auxílio de uma trena métrica que partiu da base da planta rente ao solo até o ponto de inserção.

- Número de flores;

A contagem da flor foi feita no ponto de colheita da flor obtido com as médias das três primeiras flores emergidas da haste floral.

- Massa seca da haste floral e total;

A haste floral e a parte aérea total da planta foram colhidas aos 150 DAP. Ambas foram adicionadas em sacos de papel e levados para secagem em estufa a uma temperatura de $65^{\circ} \mathrm{C}$, com circulação de ar forçado até atingirem massa constante que durou cerca de 72 horas. Após esse período, a massa seca das hastes florais e a massa seca total foram pesadas em uma balança semi-analítica.

Os resultados foram submetidos à análise a variância, e posteriormente a regressão, ambas com $5 \%$ de significância, por meio do programa estatístico SISVAR 5.6 (FERREIRA, 2011). 


\section{RESULTADOS E DISCUSSÃO}

Houve diferença significativa para as variáveis, altura de plantas, diâmetro do caule, altura da espiga, número de flores, massa de plantas secas quando submetida à diferentes doses de cinza vegetal. Resultados semelhantes também foram verificados por Bonfim - Silva et al. (2011), quando comparado ao tratamento com a ausência de cinza vegetal, para a cultura de gladíolos.

TABELA 2. Resumo da análise de variância quanto à altura de plantas (ALTP), diâmetro do caule (DC), comprimento da haste floral (HF), altura da espiga (ALTE), número de flores (NF) e massa de plantas secas (MS), do cultivo de gladíolos em resposta à adubação com cinza vegetal.

Fonte de

Variação GL QM

\begin{tabular}{|c|c|c|c|c|c|c|c|}
\hline & & $\begin{array}{l}\text { ALTP } \\
(\mathrm{cm})\end{array}$ & $\begin{array}{l}\mathrm{DC} \\
(\mathrm{cm})\end{array}$ & $\begin{array}{l}\mathrm{HF} \\
(\mathrm{cm})\end{array}$ & $\begin{array}{l}\text { ALTE } \\
(\mathrm{cm})\end{array}$ & $\begin{array}{c}\text { NF } \\
\left(n^{\circ} \text { vaso }^{-1}\right)\end{array}$ & $\begin{array}{l}\text { MS } \\
\left(\mathrm{g} / \mathrm{dm}^{-3}\right)\end{array}$ \\
\hline Tratamento & 4 & $233,72^{*}$ & $1,14^{*}$ & $6291,03^{*}$ & $355,61^{*}$ & $2,58^{*}$ & $46,36^{*}$ \\
\hline Repetição & 5 & 18,70 & 0,46 & 695,81 & 94,61 & $0,91^{*}$ & 2,91 \\
\hline Erro & 20 & 125,00 & 0,44 & 1153,51 & 146,29 & 1,46 & 10,34 \\
\hline Média & & 22,88 & 2,41 & 40,66 & 18,57 & 19,62 & 34,34 \\
\hline C.V.(\%) & & 18,57 & 27,70 & 40,66 & 37,08 & 40,93 & 34,34 \\
\hline
\end{tabular}

Em que: * é significativo a $5 \%$ de probabilidade pelo teste de $\mathrm{F}$, respectivamente.

\section{ALTURA DE PLANTAS}

A dose de cinza vegetal que proporcionou a maior altura de plantas ajustando-se ao modelo quadrático de regressão foi a dose de $11,87 \mathrm{~g} \mathrm{dm}^{-3}$, com incremento de $25,34 \%$ quando comparado com o tratamento que não recebeu a cinza (Figura 01). Isso pode ter ocorrido pois a concentração de nutrientes disponíveis no solo foram capazes de suprir a necessidade da plantas mantendo o equilíbrio sem excesso.

Bonfim-Silva et al. (2011) observaram que a altura de plantas apresentou resposta linear às doses de cinza vegetal de até $3,75 \mathrm{~g} \mathrm{dm}^{-3}$ no primeiro e no segundo corte do capim-marandu (Brachiaria brizantha). Outro trabalho que também apresentou esse efeito positivo é o de Prado et al. (2003) que ao avaliarem o efeito da cinza vegetal no substrato para produção de mudas de goiabeira, observaram incremento na altura de plantas com a aplicação da cinza até de 1,0-1,2 e 1,2-1,6 g por vaso, em solo com $\mathrm{V}=50 \%$ e $80 \%$, respectivamente. 
FIGURA 1: Altura de plantas de Gladiolus x grandiflorus em função das doses de cinza vegetal em Latossolo Vermelho do Cerrado.

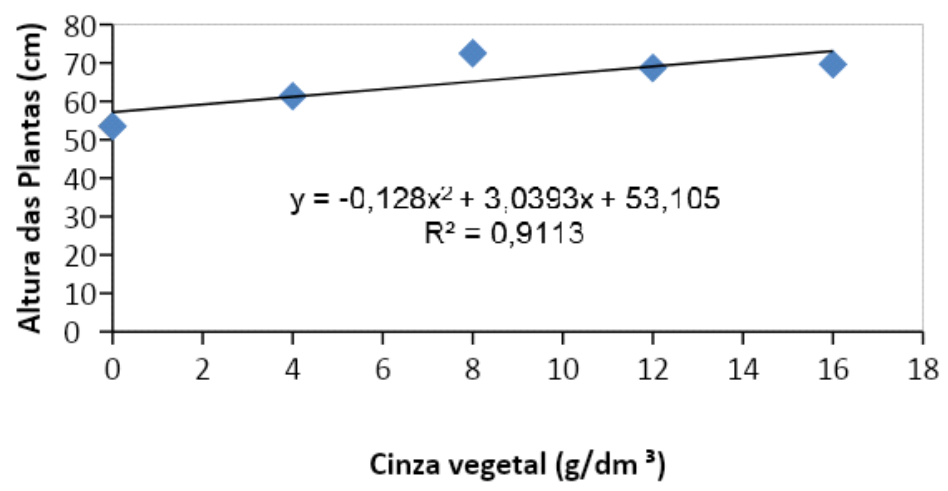

\section{DIÂMETRO DO CAULE}

A dose de cinza vegetal que proporcionou o maior diâmetro de caule de plantas ajustando-se ao modelo quadrático de regressão foi a dose de $10,63 \mathrm{~g} \mathrm{dm}^{-3}$, com incremento de $33,33 \%$ quando comparado com o tratamento que não recebeu a cinza (Figura 2). Segundo Carneiro (1995), plantas que apresentam um maior diâmetro de caule possuem maior equilíbrio no crescimento da parte aérea.

FIGURA 2: Diâmetro do caule de plantas de Gladiolus x grandiflorus em função das doses de cinza vegetal em Latossolo Vermelho do Cerrado.

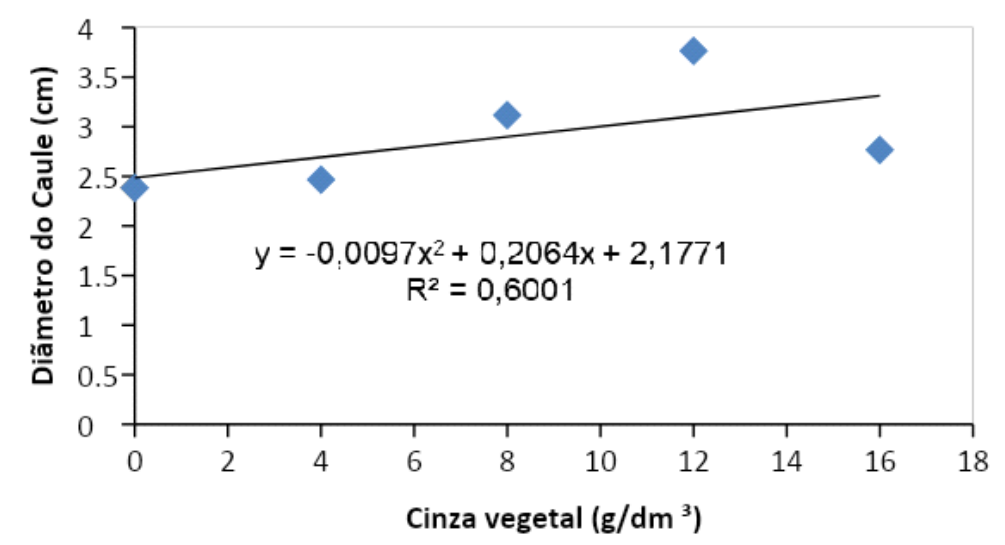

\section{COMPRIMENTO DA HASTE FLORAL}

A dose de cinza vegetal que proporcionou a maior haste floral das plantas foi de $9,30 \mathrm{~g} \mathrm{dm}^{-3}$, com incremento de $70,30 \%$ quando comparado com a dose mínima do tratamento (Figura 3). Esse aumento é uma importante característica na comercialização de flores ornamentais, favorecendo assim plantas mais chamativas ao consumidor (IBRAFLOR, 2014). Segundo Tombolato (2004), a adubação de cobertura 
nitrogenada e potássica é importante para a produção de hastes florais mais longas. Sendo assim, evidenciada a participação do potássio oriundo da cinza vegetal para o comprimento da haste floral nessas condições, tendo em vista que a adubação nitrogenada foi igual em todos os tratamentos.

FIGURA 3: Tamanho da haste floral de plantas de Gladiolus $x$ grandiflorus em função das doses de cinza vegetal em Latossolo Vermelho do Cerrado.

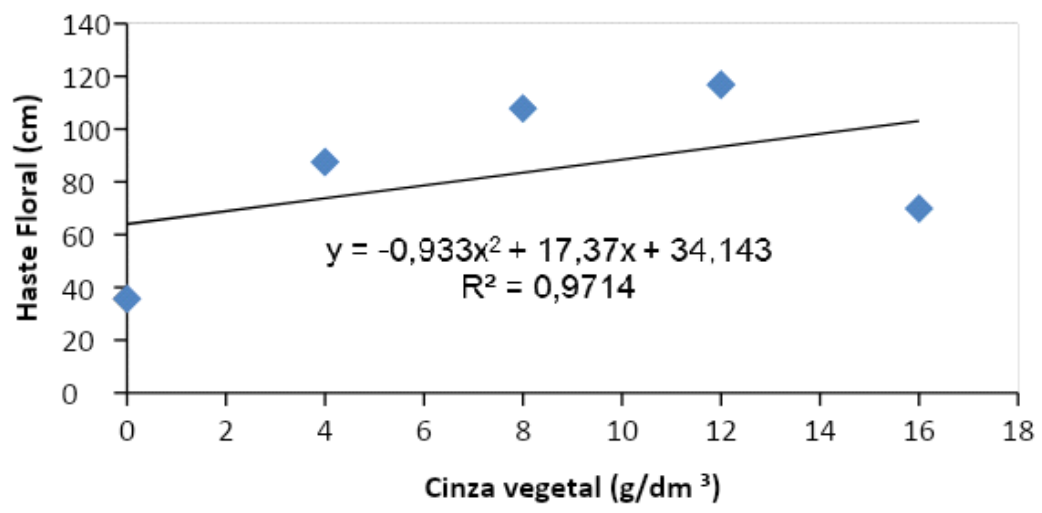

\section{ALTURA DA ESPIGA}

A dose de cinza vegetal que proporcionou a maior altura de espiga das plantas foi de $9,09 \mathrm{~g} \mathrm{dm}^{-3}$, com incremento de $73,98 \%$ quando comparado com o tratamento que não recebeu a cinza (Figura 4). De acordo com Bonfim - Silva et al. (2011) a cinza vegetal é um resíduo rico em fósforo, potássio e cálcio o que contribui para o aumento da produção. A baixa disponibilidade desses nutrientes no solo afeta as plantas reduzindo o crescimento, o que limita a captação da radiação solar e, conseqüentemente, menor produção de fotoassimilados. Isso afeta a emergência das raízes, reduzindo a capacidade de absorção de nutrientes e menor tolerância ao déficit hídrico, consequentemente reduz o crescimento da planta e assim, afetando a altura das espigas.

FIGURA 4: Altura da espiga de plantas de Gladiolus x grandiflorus em função das doses de cinza vegetal em Latossolo Vermelho do Cerrado.

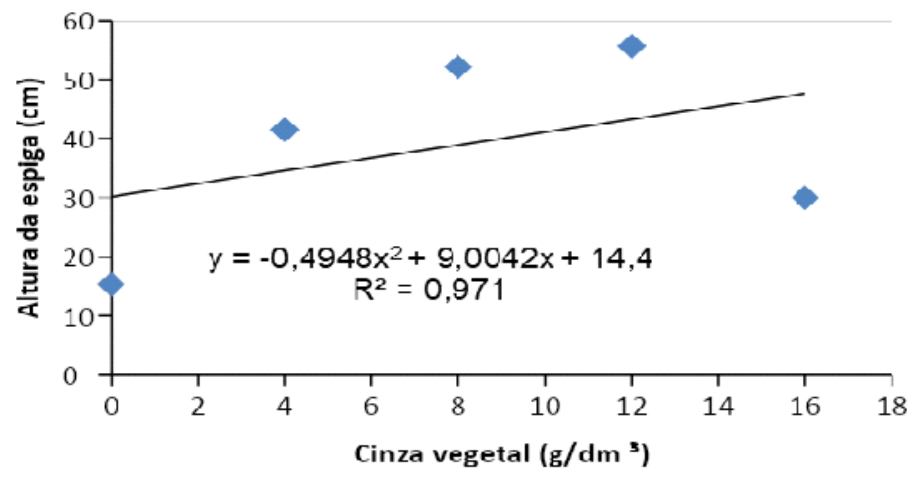




\section{NÚMERO DE FLORES}

A dose de cinza vegetal que proporcionou maior número de flores foi de $9,13 \mathrm{~g}$ $\mathrm{dm}^{-3}$, com incremento de $74,33 \%$ quando comparado com o tratamento que não recebeu a cinza (Figura 5). De acordo com Woltz (1955), o nitrogênio é responsável pelo número de hastes florais produzidas e pelo número de botões florais por haste, enquanto o potássio influencia diretamente o comprimento da haste floral. Segundo Rufty Junior et al. (1993) a redução da absorção de nitrogênio por plantas deficientes em fósforo pode ser consequência de vários fatores associados com a condição de estresse de fósforo. Uma possibilidade seria a redução da disponibilidade de energia (ATP) requerida para a absorção ativa de nitrato através da plasmalema das células radiculares.

FIGURA 5:Número de flores de Gladiolus $x$ grandiflorus em função das doses de cinza vegetal em Latossolo Vermelho do Cerrado.

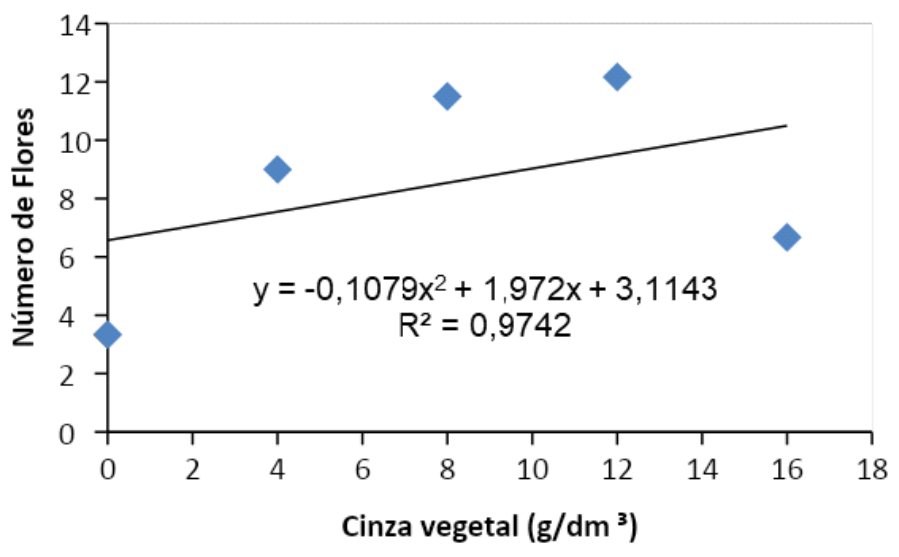

\section{MASSA SECA DE PLANTAS}

Houve diferença significativa para massa seca das plantas quanto às doses de cinza vegetal aplicadas, ajustando-se a modelo quadrático de regressão (Figura 6). A dose de cinza vegetal que proporcionou o maior incremento de massa seca das plantas foi de $9,38 \mathrm{~g} \mathrm{dm}^{-3}$, com incremento de $56,29 \%$ quando comparado com o tratamento que não recebeu a cinza.

Bonfim - Silva et al. (2011) encontraram para a máxima produção de massa seca da parte aérea de Crotalária juncea, a dose de cinza vegetal de $14,02 \mathrm{~g} \mathrm{dm}^{-3}$, proporcionando assim incremento de $66,27 \%$ na produção comparado com os resultados na ausência da adubação com cinza vegetal. A maior produção de massa seca da parte aérea foi de $11,78 \mathrm{~g}$ vaso-1. 
FIGURA 6: Massa seca de flores de Gladiolus x grandiflorus em função das doses de cinza vegetal em Latossolo Vermelho do Cerrado.

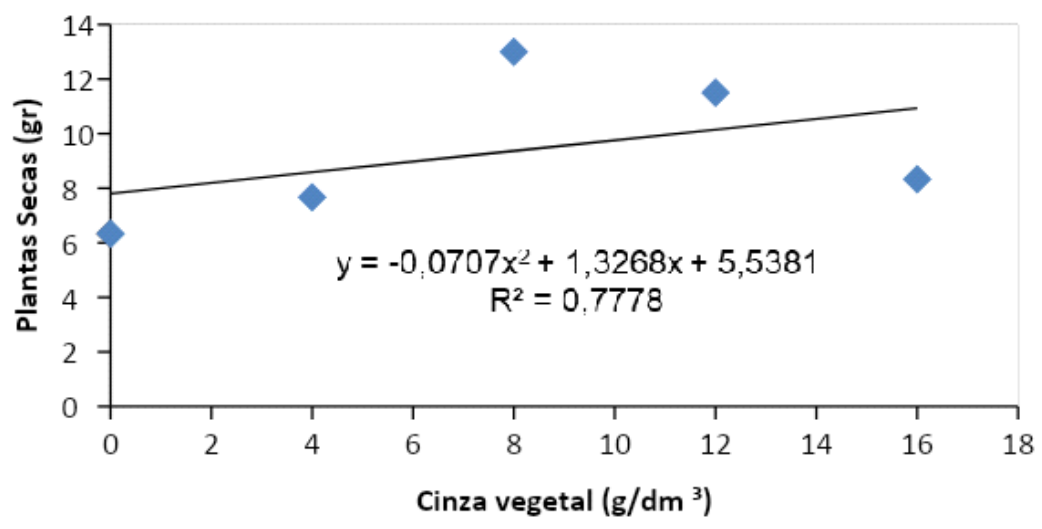

\section{CONCLUSÕES}

As plantas do Gladíolo responderam positivamente à aplicação de cinza vegetal, com um aumento na altura de plantas, diâmetro do caule, haste floral, altura da espiga, número de flores, massa de plantas secas. Para o bom desenvolvimento da cultura do gladíolos, deve - se usar uma dose entre 9 e $12 \mathrm{~g} \mathrm{dm}^{-3}$ de cinza vegetal.

\section{REFERÊNCIAS}

AHMAD, T.; AHMAD, I.; AMP; QASIM, M.; Present status and futureprospects of gladiolus cultivation in Punjab, Pakistan. Journalof Tekirdag Agricultural Faculty, v.5 p. 227-238, 2008.

AHMAD, I.; KHATTAK, A.M.; ARA, N.; AMP, AMIN NU. Effect ofplanting dates on the growth of gladiolus corms in Peshawar. Sarhad Journal of Agriculture, v.27 p. 195199, 2011.

BARBOSA, J. G. (Ed.). Palma-de-santa-rita (gladíolo): produção comercial de flores e bulbos. Viçosa, MG: Editora UFV, 2011. p. 34. IBSN: 978-85-7269-412-4.

BONFIM-SILVA, E. M.; SILVA, T. J. A.; GUIMARÃES, S. L.; POLIZEL, A. C.; Desenvolvimento e Produção de Crotalária Junce Adubada com Cinza Vegetal. Enciclopédia Biosfera, Centro Científico Conhecer - Goiânia, v.7, n.13, p.371-379, 2011.

CARNEIRO, J. G. de A.; Produção e controle de qualidade de mudas florestais. Curitiba: UFPR/FUPEF, Campos:UENF, 1995. 451p.

EMBRAPA. Empresa Brasileira de Pesquisa Agropecuária. Sistema brasileiro de classificação de solos. 3 ed. Brasília, 2013. 353p. Disponível em: < http://livimagens.sct.embrapa.br/amostras/00053080.pdf>. Acesso em: 05 Jun. 2020. 
FERREIRA, D. F.; Sisvar: a computer statistical analysis system. Ciência e Agrotecnologia. v. 35, n. 6, p.1039-1042, 2011.

GUARIZ, H. R.; PICOLI, M. H. S.; CAMPANHARO, W. A.; RODRIGUES, B. P. Uso de cinzas de fornos de cerâmica como fonte de nutrientes para aproveitamento na agricultura. In: Congresso Brasileiro de Resíduos Organicos, 1., 2009, Vitória. Anais. Vitória: Incaper, 2009.

IBRAFLOR. Veiling Holambra Flores e Plantas Ornamentais. Critério de Classificação Gérbera Pote 14. Disponível em: <http://www.infoagro.com/flores/gerbera.htm>. Acesso em: 30 de outubro de 2014.

PRADO, R. M.; CORRÊA, M. C. M.; PEREIRA, L.; CINTRA, A. C. O. e NATALES, W.; Cinza da indústria de cerâmica na produção de mudas de goiabeira, efeito no crescimento e na produção de matéria seca. Revista de Agricultura, Piracicaba, v.78, n. 1, 2003.

RUFTY, T. W.; JR; ISRAEL, D. W.; VOLK, R. J.; QIU, J.; SA, T. Phosphate regulation of nitrate assimilation in soybean.Journal of Experimental Botany, v.44, 879 - 891p., 1993.

SANTOS, C. C. Cinza vegetal como corretivo e fertilizante para os capins Marande e Xaraés. Dissertação (Mestrado) Universidade Federal de Mato Grosso, Instituto de Ciências Agrárias e Tecnológicas, Programa de Pós-Graduação em Engenharia Agrícola, Rondonópolis, 2012.

SINGH, J.P.; KUMAR, K.; KATIYAR, P.N.; Effect of zinc, iron and copper on yield parameters of gladiolus. HortFlora Research Spectrum, v.1, p. 64-68, 2012.

TOMBOLATO A. F. C.. Cultivo comercial de plantas ornamentais. Campinas: Instituto Agronômico. 2004, 211p.

WOLTZ, S. S.; Effect of Differencial Supplies of Nitrogen, Potassium and Calcium on Quality and Yield of Gladiolus. Flowers and Corms. American Society for Horticultural Sciences Proceedings, Alexandria, v.6, p.427-435, 1955.

ZIMMERMANN, S.; FREY, B. Soil respiration and microbial properties in an acid forest soil: effects of wood ash. Soil Biology \&amp; Biochemistry, Elmsford, p.1-11, 2002. 